\title{
Zur Regulation der Tetrapyrrolsynthese bei Rhodospirillum rubrum
}

\author{
G. Drews \\ Lehrstuhl für Mikrobiologie, Botanisches Institut der Universität Freiburg i. Br.
}

(Z. Naturforschg. 21 b, 1224-1229 [1966] ; eingegangen am 4. Juli 1966)

\begin{abstract}
The production of bacteriochlorophyll in anaerobic light and dark cultures is strongly inhibited by 2,6-dichlorophenol-indophenol sodium, 4-amino-2-methyl-1-naphthol-hydrochloride and methylene blue. Photophosphorylation is demonstrable $45 \mathrm{~min}$ after the start of bacteriochlorophyll synthesis. The induction of bateriochlorophyll synthesis is suppressed by mitomycin if the inhibitor is added together with the process of lowering the oxygen partial pressure. The effect of puromycin is also dependent on the time of addition. Mitomycin and puromycin have no influence on the production of coproporphyrin. In the presence of mitomycin or puromycin and $\delta$-aminolevulinic acid the synthesis of porphyrin is intensely increased but the synthesis of bacteriochlorophyll is blocked. These and other results are interpreted as indicating that control over bacteriochlorophyll synthesis occurs by a gene dependent regulation fo the biosynthesis of those enzymes which are responsible for the production of bacteriochlorophyll from protoporphyrin.
\end{abstract}

Die Biosynthese der Tetrapyrrole scheint bei allen Lebewesen in den ersten Stufen nath dem gleichen Schema zu verlaufen. Sie beginnt mit der Bildung von $\delta$-Aminolaevulinsäure (ALS) aus Glycin und Succinyl-CoA und der Kondensation von $2 \delta$-ALS-Molekülen unter Wasserabspaltung zu Porphobilinogen ${ }^{1}$. Es ist bekannt, daß die Regulation der Stoffproduktion in einer Synthesekette durch einen Rückkopplungsmechanismus zwischen End- oder Zwischenprodukt und erstem Enzym erfolgen kann. Das gilt auch für die Tetrapyrrolsynthese. Lascelles hat zeigen können, daß die Aktivität der ALS-Synthetase durch verschiedene Hämine gehemmt und die Synthese des Enzyms reprimiert wird und dadurch auch die Bacteriochlorophyll-Synthese blockiert werden kann ${ }^{1}$.

Bei verzweigten Syntheseketten, wie der Aspartatfamilie $^{3}$ und den Tetrapyrrolen ${ }^{1}$ müssen nun aber zusätzliche Mechanismen ausgebildet sein, um die Synthese der einzelnen Endprodukte unabhängig voneinander regulieren zu können.

Es gibt eine Reihe von Befunden, die zeigen, $\mathrm{da} ß$ die Bacteriochlorophyll-Synthese nicht allein auf der Stufe der ALS-Synthetase reguliert wird. So hemmt z. B. Puromycin und Chloramphenicol die

1 J. Lascelles, Tetrapyrrole, biosynthesis and its regulation. W. A. Benjamin, New York, Amsterdam 1964.

2 M. J. Bull u. J. Lascelles, Biochem. J. 87, 15 [1963].

3 L. Burlant, P. Datta u. H. Gest, Science 148, 1351 [1965].

4 G. Drews, Arch. Mikrobiol. 51, 186 [1965].

5 G. Drews u. J. Oelze, Zbl. Bakteriol. Abt. II 120, 1 [1966].

6 B. F. Burnham and J. Lascelles, Biochem. J. 87, 462 [1963].

7 G. Schön u. J. Drews, Arch. Mikrobiol. 54, 199 [1966].
B.-Chlorophyllsynthese ${ }^{2,4}$, ohne einen Einfluß auf die Bildung von Coproporphyrin zu haben ${ }^{5}$. Dieses Ergebnis wäre auch durch die Existenz von zwei oder mehreren ALS-Synthetasen mit verschiedenen allosterischen Bezirken erklärbar ${ }^{6,10}$. Nach bisherigen Untersuchungen gibt es aber keine Anhaltspunkte für die Ausbildung von Isoenzymen der ALS-Synthetase bei den Athiorhodaceen. Vielmehr sprechen viele Ergebnisse dafür, daß die Bacteriochlorophyll-Synthese auch nach der Aufzweigung der Tetrapyrrol-Syntheseketten, auf der Stufe der Holochrombildung also der Bindung an Protein gesteuert werden kann ${ }^{4,5}$.

In der vorliegenden Arbeit sollen eine Reihe von Befunden vorgelegt werden, die zusammen mit den Ergebnissen anderer Autoren sich in die Arbeitshypothese einfügen lassen, daß dieSynthese von B.-Chlorophyll aus Protoporphyrin durch Induktion und Repression eines oder mehrerer Enzyme gesteuert wird und die für die Ausbildung des Photosyntheseapparates (Thylakoide) verantwortlichen Strukturgene regulativ gekoppelt und möglicherweise zu einem Operon zusammengefaßt sind. Der wichtigste Außenfaktor, der unter natürlichen Bedingungen die Bacteriochlo-

8 G. Cohen-Bazire, W. R. Sistrom, and R. Y. Stanier, J. cellular. comparat. Physiol. 49, 25 [1957].

9 M. Biedermani, G. Drews, R. Marx u. J. Schröder, Arch. Mikrobiol., im Druck.

10 T. G. Lessie and W. R. Sistrom, Biochim. biophysica Acta [Amsterdam] 86, 250 [1964].

11 G. Drews u. P. Giesbrecht, Zbl. Bakteriol. Abt. I 190, 508 [1963].

12 S. P. Gibbs, W. R. Sistrom, and P. B. Worden, J. Cell. Biol. 26, 395 [1965]; S. C. Holt and A. G. Marr, J. Bacteriol. 89, 1421 [1965]. 
rophyll-Synthese reguliert, ist der Sauerstoffpartialdruck im Medium 1, 6, 7, 9, 13, und in gewissem Umfange auch das Licht ${ }^{14}$. Die Ergebnisse einer gesonderten Mitteilung über die quantitativen Beziehungen zwischen $\mathrm{O}_{2}$-Partialdruck und B.-Chlorophyllbildung zeigen, daß unterhalb eines relativ scharf ausgebildeten Schwellenwertes eine Umschaltung in der Zelle erfolgt ${ }^{9}$. Wahrscheinlich wirkt das Folgeprodukt einer Redoxreaktion aktivierend auf die Gene, etwa in der Art, daß ein spezifisches Molekül den Repressor inaktiviert. Wir kennen aber den Mechanismus nicht und wissen nur, daß indirekt über den Sauerstoffpartialdruck die Thylakoidbildung als ein genabhängiger Prozeß gesteuert wird ${ }^{4,5}$. Unsere Untersuchungen haben auch ergeben, daß eine Synthese spezifischer Enzyme für die B.-Chlorophyllbildung entweder unter semiaeroben Bedingungen stattfindet, wenn also die Enzymbildung induziert ist und der $\mathrm{O}_{2}$-Partialdruck noch ausreicht, um eine oxydative Phosphorylierung zu ermöglichen, oder aber unter streng anaeroben Bedingungen, wenn Licht und ein ausgebildetes Thylakoidsystem Photophosphorylierung ermöglichen ${ }^{7,9}$.

Wenn man der anaeroben Kultur reduzierbare Verbindungen zusetzt, und damit das Redoxgleichgewicht in dem für die Induktion der B.-Chlorophyllbildung verantwortlichen System verschiebt, wird die B.-Chlorophyllsynthese gehemmt (Tab. 1). $\mathrm{KNO}_{3}$, das von $R$. rubrum nicht reduziert werden kann, hat keinen Einfluß. Dagegen wirken Methylenblau und Dichlorophenolindophenol deutlich hemmend. Menadionbisulfit hat den gleichen Wirkungsgrad wie 4Amino-2-methyl-1-naphtolhydrochlorid.

Die Enzyme des ersten Abschnittes der B.-Chlorophyllsynthese, die für die Synthese aller Tetrapyrrole benötigt werden, sind konstitutiv ${ }^{1,4,5}$. Die Regulation der gesamten Tetrapyrrolproduktion erfolgt hauptsächlich durch eine Steuerung der Aktivität der ALS-Synthetase ${ }^{1}$, in gewissem Umfange aber auch durch unterschiedliche Enzymproduktion 4, 5, 6 . Es war aber bislang nicht bekannt, ob die für den 2. Abschnitt der B.-Chlorophyllsynthese und die Thylakoidbildung spezifischen Enzyme auch in aeroben Dunkelkulturen, also bei Fehlen des Photosyntheseapparates bereits vorhanden sind, oder erst durch Induktion gebildet werden. Die Kinetik der

13 W. R. Sistrom, J. Bacteriol. 89, 403 [1965].

14 M. Higuchi, K. Goto, M. Fugrmoto, O. Namikiand, G. Kıкисні, Biochim. biophysica Acta [Amsterdam] 95, 94 [1965].

\begin{tabular}{|c|c|c|c|c|}
\hline $\begin{array}{c}\text { Art und } \\
\text { Dauer der } \\
\text { Bebrütung } \\
\text { [Stdn.] }\end{array}$ & $\begin{array}{c}\text { Zusätze } \\
\text { zur } \\
\text { Nährlösung }\end{array}$ & $\begin{array}{c}\text { B.- } \\
\text { Chloro- } \\
\text { phyll } \\
{[\mu \mathrm{g} / \mathrm{ml}]}\end{array}$ & $\begin{array}{l}\text { Protein } \\
{[\mu \mathrm{g} / \mathrm{ml}]}\end{array}$ & $\begin{array}{c}\text { B.- } \\
\text { Chloro- } \\
\text { phyll } \\
\text { pro } \\
\text { Protein } \\
{[\mu \mathrm{g} / \mathrm{mg}]}\end{array}$ \\
\hline $\begin{array}{l}\text { Dun el, } 2 \\
\text { dunkel, } 3\end{array}$ & $\begin{array}{l}\text { DCPIP } \\
10^{-4}-m . \\
\text { Vitamin K } 5 \\
10^{-4}-m . \\
\quad- \\
\mathrm{KNO}_{3} 1,0 \% \\
\text { DCPIP } \\
\mathrm{K} 5 \\
\text { Methylen- } \\
\text { blau } 10^{-4}-m .\end{array}$ & $\begin{array}{l}0,575 \\
0,355 \\
\\
0,370 \\
0,760 \\
0,750 \\
0,400 \\
0,350 \\
0,283\end{array}$ & $\begin{array}{l}268 \\
277 \\
\\
257 \\
282 \\
273 \\
261 \\
256 \\
\\
296\end{array}$ & $\begin{array}{l}2,15 \\
1,28 \\
1,44 \\
2,70 \\
2,75 \\
1,53 \\
1,36 \\
0,95\end{array}$ \\
\hline $\begin{array}{l}\text { dunkel, } 3 \\
\text { dann Licht, } 3\end{array}$ & \begin{tabular}{l}
\multicolumn{1}{c}{-} \\
$\mathrm{KNO}_{3}$ \\
$\mathrm{DCPIP}$ \\
$\mathrm{K} 5$ \\
Methylen- \\
blau
\end{tabular} & $\begin{array}{l}2,80 \\
2,84 \\
0,53 \\
0,40 \\
\\
0,34\end{array}$ & $\begin{array}{l}267 \\
259 \\
257 \\
246 \\
\\
274\end{array}$ & $\begin{array}{r}10,50 \\
11,00 \\
2,06 \\
1,63 \\
\\
1,24\end{array}$ \\
\hline $\begin{array}{l}\text { dunkel, } 3 \\
\text { dann Licht, } 21\end{array}$ & $\begin{array}{l}\mathrm{KNO}_{3}^{-} \\
\mathrm{K} 5\end{array}$ & $\begin{array}{c}53,8 \\
59,1 \\
0,74\end{array}$ & $\begin{array}{l}635 \\
700 \\
657\end{array}$ & $\begin{array}{c}84,5 \\
84,3 \\
1,12\end{array}$ \\
\hline
\end{tabular}

Tab. 1. Die Einwirkung von verschiedenen Substanzen auf die Bildung von Bacteriochlorophyll in anaeroben Kulturen von Rhodospirillum rubrum. Vorkultur: aerob, dunkel. Temperatur: $30^{\circ} \mathrm{C}$. DCPIP: 2.6-Dichlorphenolindophenol Natriumsalz, K5 =4-Amino-2-methyl-1-naphtol-hydrochlorid. Die Methodik für die Anzucht der Organismen sowie die analy. tischen Bestimmungen sind in l. c. ${ }^{4,5,7}$ beschrieben.

B.-Chlorophyllsynthese in Dunkelkulturen nach raschem Absenken des $\mathrm{O}_{2}$-Partialdruckes spricht dafür, daß geringe Mengen der Enzyme auch in Zellen aus aeroben Dunkelkulturen enthalten sind ${ }^{7}$, so daß eine mehrstündige konstante Pigmentproduktion möglich ist, deren Dauer von der in Reservestoffen gespeicherten Energie abhängt ${ }^{7}$. Die Enzymmenge muß aber sehr gering sein und der Anstieg des Enzymspiegels in wachsenden anaeroben Lichtkulturen um ein Vielfaches größer sein als z. B. die Zunahme der ALS-Synthetase unter gleichen Bedingungen ${ }^{4,5}$. Die Untersuchung der Regulation wird noch dadurch kompliziert, daß die Enzymneubildung nach Induktion nur stattfinden kann, wenn dem System Energie durch Photophosphorylierung oder durch oxydative Phosphorylierung in "semiaeroben “ Dunkelkulturen zufließt. Wenn die Absenkung des $\mathrm{O}_{2}$-Partialdruckes sehr rasch erfolgt, z. B. durch Begasen mit gereinigtem Helium, und im Dunkeln bebrütet wird, ist die B.-Chlorophyllsynthese nur sehr gering $(0,1 \mu \mathrm{g} / \mathrm{mg}$ Protein/Stde.). 
Die Photophosphorylierung läßt sich bereits nach $45 \mathrm{~min}$ anaerober Lichtkultur nachweisen (Tab. 2) und bedingt in anaeroben Lichtkulturen eine starke Zunahme der B.-Chlorophyllproduktionsrate nach etwa 90 Minuten ${ }^{7}$.

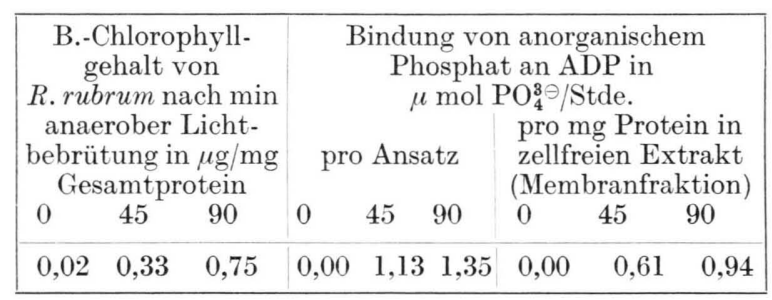

Tab. 2. Photophosphorylierung in zellfreien Extrakten von R. rubrum nach verschiedenen Zeiten anaerober Vorbebrütung im Licht. Methodik s. 1. c. ${ }^{7}$.

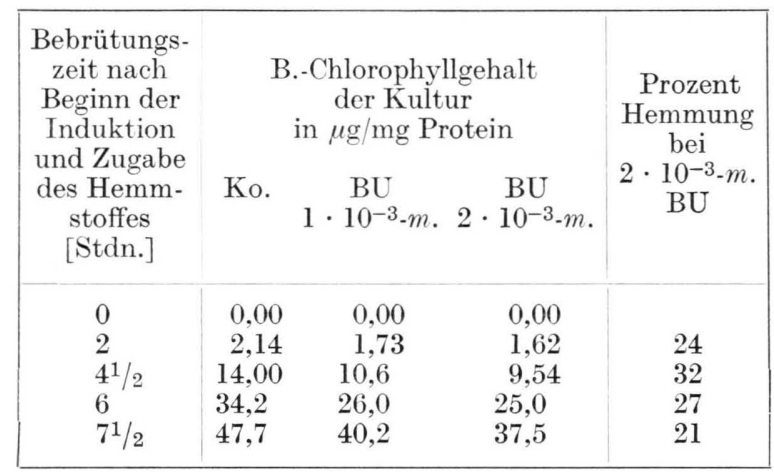

Tab. 3. Hemmung der B.-Chlorophyllbildung durch Bromuracil (BU).

Wenn die Bacteriochlorophyll- und Thylakoidbildung durch Repression und Derepression der "Thylakoid-Gene" reguliert wird, müßte ein Eingriff in den Nucleinsäurestoffwechsel die B.Chlorophyllsynthese spezifisch beeinflussen. Wie die Tabn. 3 und 4 a zeigen, beträgt die spezifische Hemmung der B.-Chlorophyllbildung durch Bromuracil $\left(2 \cdot 10^{-3}-m\right.$.) etwa $25 \%$, durch Mitomycin ${ }^{4,14}$ $\left(7 \cdot 10^{-6}-m\right.$.) etwa $75 \%$ im Zeitraum von 8 Stunden. Fluordesoxyuridin $(150 \mu \mathrm{g} / \mathrm{ml})$ hemmt nach 4,6 und $22 \mathrm{~h}$ etwa 10 Prozent. Wenn Mitomycin Kulturen zugesetzt wird, die nicht aerob, sondern anaerob vorbebrütet wurden (Tabn. $4 \mathrm{a}, \mathrm{B}$ ), dann ist der Grad der Hemmung zunächst sehr gering (7\%), weil der Syntheseapparat schon ausgebildet ist, steigt aber nach 8 Stdn. auf 32 und nach 24 Stdn. auf $40 \%$ an. Diese Hemmungszunahme dürfte dadurch bedingt sein, daß nach Blockierung der NS Synthese keine Neusynthese an Enzymmolekülen statt- findet und - in Abhängigkeit von der Lebensdauer der einzelnen Enzyme - ihr Gehalt pro Zelle abnimmt.

Wenn anaerobe Lichtkulturen verdünnt werden, so erhöht sich der Anteil der Lichtquanten, der von der einzelnen Zelle absorbiert wird. Dadurch wird ein Regulationsmechanismus ausgelöst, der für einige Zeit die B.-Chlorophyllsyntheserate vermindert (Tabn. 4a, b). Um diesen Effekt auszuschließen,

\begin{tabular}{|c|c|c|c|c|c|c|}
\hline \multirow{3}{*}{$\begin{array}{l}\text { Bebrütungs- } \\
\text { zeit nach } \\
\text { Zugabe von } \\
\text { Mitomycin } \\
\text { [Stdn.] }\end{array}$} & \multicolumn{4}{|c|}{$\begin{array}{l}\text { B.-Chlorophyllgehalt der } \\
\text { Kultur in } \mu \mathrm{g} / \mathrm{mg} \text { Protein }\end{array}$} & \multirow{2}{*}{\multicolumn{2}{|c|}{$\begin{array}{l}\text { Hemmung } \\
\text { gegenüber } \\
\text { der Kontrolle } \\
\text { in Prozent }\end{array}$}} \\
\hline & \multicolumn{2}{|c|}{ Kultur A } & \multicolumn{2}{|c|}{ Kultur B } & & \\
\hline & Ko. & Mi. & Ko. & Mi. & $\mathrm{A}$ & B \\
\hline 0 & 0,4 & 0,4 & 69,0 & 69,0 & & \\
\hline 2 & 5,3 & 2,00 & 66,2 & 61,5 & 62 & 7 \\
\hline 4 & 8,8 & 2,38 & 64,5 & 55,0 & 73 & 15 \\
\hline 8 & 31,8 & 3,74 & 82,0 & 55,5 & 88 & 32 \\
\hline 24 & 80,4 & 2,11 & 89,0 & 53,5 & 98 & 40 \\
\hline
\end{tabular}

Tab. 4 a. Hemmung der B-Chlorophyllbildung durch Mitomycin $(2,6 \mu \mathrm{g} / \mathrm{ml})$. Alle Kulturen werden anaerob im Licht bei $30{ }^{\circ} \mathrm{C}$ bebrütet. Vorkultur A: $21 \mathrm{Stdn}$. aerob, dunkel. B : 21 Stdn. anaerob. Licht. Beide Kulturen wurden nach der Vorbebrütung mit frischer Nährlösung verdünnt.

\begin{tabular}{|c|c|c|c|c|c|c|}
\hline $\begin{array}{l}\text { Bebrütungs- } \\
\text { zeit nach } \\
\text { Zugabe von } \\
\text { Mitomycin } \\
\text { [Stdn.] }\end{array}$ & \multicolumn{2}{|c|}{$\begin{array}{l}\text { B.-Chloro- } \\
\text { phyll } \\
{[\mu \mathrm{g} / \mathrm{ml}]}\end{array}$} & \multicolumn{2}{|c|}{$\begin{array}{l}\text { Protein } \\
{[\mu \mathrm{g} / \mathrm{ml}]}\end{array}$} & \multicolumn{2}{|c|}{$\begin{array}{c}\text { B.-Chloro- } \\
\text { phyll/Protein } \\
{[\mu \mathrm{g} / \mathrm{mg}]}\end{array}$} \\
\hline 0 & $\begin{array}{l}\text { Ko. } \\
14,2\end{array}$ & $\begin{array}{c}\text { Mi. } \\
12,6\end{array}$ & $\begin{array}{l}\text { Ko. } \\
242\end{array}$ & $\begin{array}{l}\text { Mi. } \\
238\end{array}$ & $\begin{array}{l}\text { Ko. } \\
58,8\end{array}$ & $\begin{array}{l}\text { Mi. } \\
52,8\end{array}$ \\
\hline 1 & 16,3 & 14 & 248 & 25 & 65,8 & 57,0 \\
\hline 2 & 18,8 & 15, & 264 & 26 & 71,3 & 59,0 \\
\hline 3 & 20,7 & 17 & 278 & 27 & 74,6 & 63,0 \\
\hline 5 & 26,5 & 18 & 340 & 30 & 78,0 & 61,4 \\
\hline 7 & 30,0 & 18 & 362 & 312 & 83,0 & 60,0 \\
\hline
\end{tabular}

Tab. 4 b. Hemmung der B.-Chlorophyllsynthese in anaeroben Lichtkulturen mit Mitomycin $(4,0 \mu \mathrm{g} / \mathrm{ml})$. Vorkultur: 14 Stdn. anaerob im Licht $\left(30^{\circ} \mathrm{C}, 2200 \mathrm{Lux}\right)$.

wurde in dem in Tab. $4 \mathrm{~b}$ wiedergegebenen Versuch der Hemmstoff ohne die Zelldichte zu verändern zugesetzt. Unter diesen Bedingungen führt Mitomycin nach $3 \mathrm{Stdn}$. zu einer vollständigen Sistierung der B.-Chlorophyllsynthese, während das Wachstum andauert. Der B.-Chlorophyllgehalt der Zellen nimmt also ab. Wir kennen nicht die Lebensdauer des Enzyms, aber die der m-RNS muß auf jeden Fall geringer als 3 Stdn. sein. Wie wir aus Tab. 4 c sehen, bewirkt Puromycin unter den gleichen Versuchsbedingungen eine vollständige Hemmung der Pigmentsynthese nach 6 Stunden. Von dieser Zeit ab 


\begin{tabular}{|c|cc|cc|cc|}
\hline $\begin{array}{c}\text { Bebrütungs- } \\
\text { zeit nach } \\
\begin{array}{c}\text { Zugabe von } \\
\text { Puromycin } \\
\text { [Stdn. }\end{array}\end{array}$ & $\begin{array}{c}\text { B.-Chloro- } \\
\text { phyll }\end{array}$ & Protein & $\begin{array}{c}\text { B.-Chloro- } \\
\text { phyll/Protein }\end{array}$ \\
\hline & {$[\mu \mathrm{g} / \mathrm{ml}]$} & \multicolumn{2}{|c|}{$[\mu \mathrm{g} / \mathrm{ml}]$} & \multicolumn{2}{|c|}{$[\mu \mathrm{g} / \mathrm{mg}]$} \\
\hline 0 & Ko. & $\mathrm{Pu}$. & Ko. & $\mathrm{Pu}$. & Ko. & $\mathrm{Pu}$. \\
2 & 7,02 & 5,90 & 152 & 130 & 46,3 & 45,4 \\
4,5 & 10,41 & 8,00 & 172 & 152 & 60,5 & 52,5 \\
6 & 16,98 & 10,18 & 230 & 182 & 73,9 & 56,0 \\
7 & 21,8 & 11,15 & 261 & 206 & 83,9 & 54,1 \\
8,5 & 24,7 & 10,6 & 332 & 254 & 74,5 & 41,7 \\
\hline
\end{tabular}

Tab. 4 c. Einfluß von Puromycin auf die B.-Chlorophyllbildung in anaeroben Lichtkulturen. Vorbebrütung: $13 \mathrm{Stdn}$. anaerob im Licht. Dann Zugabe von Puromycin $(8 \mu \mathrm{g} / \mathrm{ml})$.

wird kein B.-Chlorophyll mehr synthetisiert, d.h. es sind keine aktiven Enzymmoleküle mehr vorhanden, obwohl das Wachstum noch andauert. Der B.-Chlorophyllgehalt der Zellen nimmt schon nach $4^{1 / 2}$ Stdn. ab. Aus der Keimzahlbestimmung und der Proteinzunahme folgt, daß in wachsenden Zellen die Lebensdauer dieser Enzymmoleküle geringer als die Generationszeit sein muß.

Wenn man das Produkt des 1. Enzyms der Tetrapyrrolsynthesekette, die $\delta$-Aminolaevulinsäure, dem Ansatz zufügt, so ist der Effekt auf die B.-Chlorophyllsynthese relativ gering. In den ersten Stdn. nach Induktion beobachtet man eine schwache Hem. mung, nach längerer Bebrütung (24 Stdn.) allgemein eine Förderung weil bei Erschöpfung des Nährbodens die ALS direkt in die Pigmentsynthese eingeschleust werden kann (Tab. 5). Dagegen wird die Bildung von Coproporphyrin in höherem Maße gefördert. Dieser Effekt wird noch erheblich verstärkt, wenn die B.-Chlorophyllproduktion durch Mitomycin gestoppt wurde (Tab. 5). Wir können aus diesen Experimenten entnehmen, daß die Enzyme der Tetrapyrrolsynthese bis zum Coproporphyrin konstitutiv sind und eine große Lebensdauer besitzen oder eine langlebige m-RNS, so daß Mitomycin nicht hemmt. Die B.-Chlorophyllbildung dagegen wird unabhän- gig von der Porphyrinsynthese reguliert und ist mit anderen Prozessen gekoppelt. Die dazu notwendigen Enzyme müssen erst induktiv gebildet werden.

Eine induktive Bildung derjenigen Enzyme, die die Synthese von B.-Chlorophyll aus Porphyrin katalysieren, läßt sich auch aus Puromycinversuchen ableiten. Wie Abb. 1 zeigt, wird kaum B.-Chlorophyll synthetisiert, wenn man Puromycin zu Beginn der anaeroben Bebrütung zufügt. Die Hemmung ist jedoch wesentlich schwächer, wenn die Bakterien erst nach 1- bzw. 3-stdg. anaerober Bebrütung mit

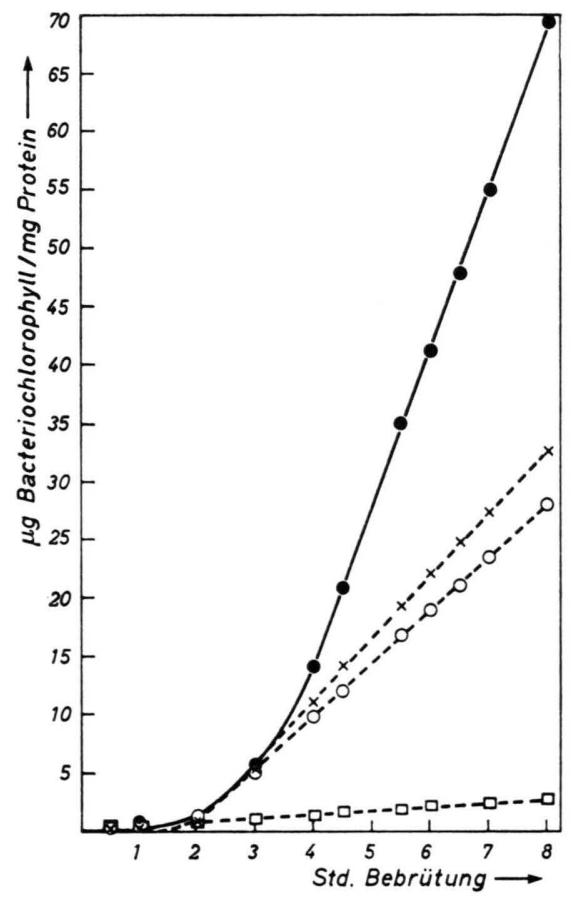

Abb. 1. Hemmung der Bacteriochlorophyll-Synthese in anaeroben Lichtkulturen von $R$. rubrum durch Puromycin $(7 \mu \mathrm{g} / \mathrm{ml})$. Temperatur: $30^{\circ} \mathrm{C}$. Kontrolle: $-\bullet$, Zugabe von Puromycin zu Beginn der Induktion (0 Stdn.). $\square . . . \square, \mathrm{Zu}-$ gabe von Puromycin 1 Stde. nach Beginn der Induktion. $0 . . .0$, Zugabe von Puromycin $3 \mathrm{Stdn}$. nach Beginn der Induktion. $+\cdots+(10 \mu \mathrm{g}$ Puromycin/ml). Vorkultur: Aerob, dunkel, Schütteltisch, $30^{\circ} \mathrm{C}$.

\begin{tabular}{|c|c|c|c|c|c|c|c|c|c|c|c|c|c|c|c|c|}
\hline \multirow{2}{*}{$\begin{array}{c}\text { Be- } \\
\text { brütungs- } \\
\text { zeit } \\
\text { [Stdn.] }\end{array}$} & \multicolumn{8}{|c|}{$\begin{array}{l}\text { B.-Chlorophyllgehalt der Kultur } \\
\text { [ } \mu \mathrm{g} / \text { B.-Chl./mg Protein }]\end{array}$} & \multicolumn{8}{|c|}{$\begin{array}{l}\text { Coproporphyringehalt der Kultur } \\
\text { [ } \mu \mathrm{g} \text { Proph./mg Protein] }\end{array}$} \\
\hline & Ko. & $\begin{array}{l}\text { Kult } \\
\text { ALS }\end{array}$ & $\begin{array}{l}\text { ur A } \\
\text { Mi. }\end{array}$ & $\begin{array}{l}\text { Mi. }+ \\
\text { ALS }\end{array}$ & Ko. & $\begin{array}{l}\text { Kult } \\
\text { ALS }\end{array}$ & $\begin{array}{l}\text { ur B B } \\
\text { Mi. }\end{array}$ & $\begin{array}{l}\text { Mi. }+ \\
\text { ALS }\end{array}$ & Ko. & $\stackrel{\mathrm{K}}{\mathrm{ALS}}$ & $\begin{array}{l}\text { ultur } \\
\text { Mi. }\end{array}$ & $\begin{array}{l}\text { Mi. }+ \\
\text { ALS }\end{array}$ & Ko. & $\begin{array}{r}\mathrm{K} \\
\mathrm{ALS}\end{array}$ & $\begin{array}{l}\text { ultur } \\
\text { Mi. }\end{array}$ & $\begin{array}{l}\mathrm{B} \\
\mathrm{Mi} .+ \\
\mathrm{ALS}\end{array}$ \\
\hline $\begin{array}{l}0 \\
4\end{array}$ & $\begin{array}{l}0,00 \\
5,44\end{array}$ & $\overline{3.88}$ & $2 \overline{72}$ & $\overline{0.09}$ & & & & & $\begin{array}{l}1,04 \\
07\end{array}$ & $\overline{1-26}$ & $\overline{122}$ & & & & & \\
\hline 7 & 33,1 & 17,1 & 2,8 & 0,0 & 75,4 & 74,5 & 47,5 & 58, & 1,11 & 1,51 & 1,66 & 2,71 & 0,48 & 1,26 & 0,47 & 2,06 \\
\hline 24 & 80,4 & 84,0 & 2,1 & 0,80 & 89,0 & 119,8 & 53,5 & 64,5 & 0,75 & 2,57 & 1,40 & 4,13 & 0,36 & 1,22 & 1,09 & 6,88 \\
\hline
\end{tabular}

Tab. 5. Bildung von Bacteriochlorophyll unter Einwirkung von Mitomycin $(2,6 \mu \mathrm{g} / \mathrm{ml}$ ) und $\delta$-Aminolaevulinsäure (ALS, $2 \cdot 10^{-3}-m$.) in anaeroben Lichtkulturen von $R$. rubrum nach aerober (A) oder anaerober (B) Vorbebrütung. 
dem Puromycin in Berührung kommen. In dieser Zeit muß die Derepression der Enzymsynthese im wesentlichen beendet sein, denn die Abnahme der Hemmung nach dieser Zeit ist geringer als zwischen 0 und 1 Stunde. Wie wir wissen, setzt die B.-Chlorophyllsynthese in anaeroben Kulturen sofort ein, weil eine geringe Enzymmenge auch in Dunkelkulturen vorhanden ist oder während der semiaeroben Phase gebildet wurde. Die ausgeprägte lag-Phase (Abb. 1) ist teilweise durch die erst nach etwa 45 min einsetzende Photophosphorylierung bedingt, die das notwendige ATP für Synthesen zur Verfügung stellt. Die Kultur, die sofort Puromycin erhielt, kann von dem steigenden Energieangebot kaum profitieren, weil sie nur die in der aeroben Kultur enthaltene oder während einer kurzen semiaeroben Phase induzierte Enzymmenge besitzt, und eine Neusynthese durch Puromycin verhindert wird. Parallel zur Ausbildung des Thylakoidapparates steigt in den Kulturen ohne Puromycin die absolute Photophosphorylierungsrate $(\mu \mathrm{Mol} \mathrm{ATP} /$ Zelle/Stde). Nach der lagPhase, deren Dauer von den Kulturbedingungen abhängig ist, stellt sich ein Gleichgewichtszustand ein, der dadurch charakterisiert ist, daß B.-Chlorophyll bezogen auf Gesamtprotein pro Zeiteinheit um einen konstanten Wert zunimmt (Fig. 1). Das Wachstum gemessen als Zunahme der optischen Dichte in der Suspension und des Gesamtproteins ( $\mu$ g Protein/ $\mathrm{ml}$ ), beginnt in dem in Fig. 1 wiedergegebenen Experiment nach etwa $5 \mathrm{Stdn}$., in anderen Versuchen meist schon nach 4 Stunden. Diese Phase der linearen Zunahme von BChl/Protein nimmt nur einen Teil der logarithmischen Wachstumsphase ein. Später erreicht der Quotient BChl/Protein also der B.-Chlorophyllgehalt pro Zelle einen konstanten Wert, der von der Lichtintensität in der Kultur abhängt ${ }^{11,12}$. Die Lichtverhältnisse in einer logarithmisch wachsenden batch-Kultur sind an sich nie konstant. Es ist aber bekannt, daß der spezifische B.-Chlorophyllgehalt der Zellen (B.-Chlorophyll/Thylakoidprotein) innerhalb eines großen Bereiches der Quantenstromdichte gleich ist ${ }^{11,12}$. Die konstante Syntheserate spricht dafür, daß die Zellen eine konstante Enzymmenge enthalten. Die Lebensdauer der Enzyme läßt sich aus den Versuchen, die sofort Puromycin erhielten (Fig. 1) oder die unter streng anaeroben Bedingungen im Dunkeln durchgeführt wurden, nicht berechnen, weil in diesen Kulturen keine Proteinsynthese nachzuweisen ist und daher auch der Enzymabbau gehemmt sein kann.
In den Kulturen, die nach 1 bzw. 3 Stdn. mit Puromycin versetzt wurden, wird das Wachstum nicht vollständig gehemmt. Es beginnt, wie in der Kontrolle, nach etwa $4^{1} / 2$ Stunden. Da die B.-Chlorophyllbildung auch in diesen Kulturen linear ansteigt, muß eine Neusynthese von Enzymprotein stattfinden. Für die Höhe der B.-Chlorophyllproduktion ist also entscheidend, ob die Hemmung zu Beginn der Induktion oder erst später einsetzt. Puromycin, das erst nach $3 \mathrm{Stdn}$. in die Kultur gegeben wird, hemmt in erster Linie das Wachstum, also auch die Neubildung des Proteinsyntheseapparates und die Synthese von Thylakoid-Strukturprotein. Die Porphyrinbildung wird durch Puromycin nicht gehemmt (Tab. 6). Die notwendigen Enzyme sind also auch

\begin{tabular}{|c|c|c|c|c|c|c|}
\hline \multirow{2}{*}{$\begin{array}{l}\text { Bebrütungs- } \\
\text { zeit nach } \\
\text { Beginn der } \\
\text { Induktion } \\
\text { [Stdn.] }\end{array}$} & \multicolumn{3}{|c|}{$\begin{array}{c}\text { B.-Chloro- } \\
\text { phyll/Protein } \\
{[\mu \mathrm{g} / \mathrm{mg}]}\end{array}$} & \multicolumn{3}{|c|}{$\begin{array}{c}\text { Copro- } \\
\text { porphyrin/Protein } \\
{[\mu \mathrm{g} / \mathrm{mg}]}\end{array}$} \\
\hline & Ko. & $\mathrm{Pu}$ & $\begin{array}{c}\mathrm{Pu}+ \\
\mathrm{ALS}\end{array}$ & Ко. & $\mathrm{Pu}$ & $\begin{array}{c}\mathrm{Pu}+ \\
\mathrm{ALS}\end{array}$ \\
\hline 4 & 3,18 & 2,85 & 1,61 & 0,69 & 0,74 & 1,07 \\
\hline 7 & 16,2 & 3,78 & 2,95 & 0,73 & 0,80 & 1,14 \\
\hline 24 & 80,0 & 4,56 & 5,48 & 0,61 & 1,66 & 2,18 \\
\hline
\end{tabular}

Tab. 6. Einfluß von Puromycin (Pu.) und $\delta$-Aminolaevulinsäure (ALS) auf die Synthese von Bacteriochlorophyll und Coproporphyrin in anaeroben Lichtkulturen von $R$. rubrum. Vorkultur: aerob, dunkel.

in aeroben Dunkelkulturen vorhanden. Wenn durch Blockierung der Proteinsynthese der pool an niedermolekularen Substanzen nicht zum Wachsen verbraucht wird, können relativ mehr Bausteine in die Porphyrinproduktion geleitet werden, so daß der Porphyrinspiegel bezogen auf Protein ansteigt (Tab. 6). Dieser Effekt wird noch durch ALS verstärkt. Da ein großer Teil des Coproporphyrins ausgeschieden werden kann, scheinen diese Substanzen keine feedback Hemmung auszulösen.

Von mehreren Autoren konnte nachgewiesen werden, daß B.-Chlorophyll- und Thylakoidstrukturprotein-Synthese gekoppelt sind ${ }^{2,4,6,8}$. In unseren Kulturen beobachteten wir aber während der lag-Phase keine nachweisbare Bruttoproduktion an Protein, obwohl die B.-Chlorophyllmenge nach Induktion sofort ansteigt. Vorläufige Befunde unserer elektronenmikroskopischen Untersuchungen zeigen, daß die ersten Thylakoidbläschen, die durch Invagination aus der cytoplasmatischen Membran von $R$. rubrum abgefaltet werden, erst nach 4 Stdn. deutlich und 
nach 2 Stdn. nur in einzelnen Zellen nachzuweisen sind. Es ist also nicht auszuschließen, daß das erste B.-Chlorophyll in die cytoplasmatische Membran eingebaut wird. Zellfraktionierungsversuche deuten noch eine andere Möglichkeit an. Zellen von $R$. rubrum wurden 4 Stdn. nach Beginn der Induktion mit der French pressure cell homogenisiert und die zellfreien Extrakte durch Zentrifugation fraktioniert. Verglichen mit 15-h-Kontrollkulturen war der B.-Chlorophyllgehalt in der Thylakoidfraktion (30 min, $90000 \mathrm{~g}, 35000 \mathrm{U} / \mathrm{min}$, Rotor 50, Spinco L 2) relativ gering (3,77 $\mu \mathrm{g}$ B.-Chlorophyll/mg Protein). Ein Teil der chlorophyllhaltigen Partikel ließ sich erst bei $150000 \mathrm{~g}(45000 \mathrm{U} / \mathrm{min}, 2 \mathrm{Stdn}$.) sedimentieren. Der B.-Chlorophyllgehalt dieser Fraktion betrug $6,18 \mu \mathrm{g} / \mathrm{mg}$ Protein. Es wäre daher auch denkbar, daß zunächst in der Zelle b.-chlorophyllhaltige Untereinheiten der Thylakoide gebildet und später in die Membranen eingebaut werden.

Der von Lascelles ${ }^{1}$ für die Tetrapyrrolsynthese postulierte Regelmechanismus durch Endprodukthemmung und Repression der ALS-Synthetase scheint zumindest für die Bacteriochlorophyll-Bildung in der lebenden Zelle von untergeordneter Bedeutung zu sein. Die hinter dem Protoporphyrin von den übrigen Tetrapyrrolen abzweigende B.Chlorophyll-Synthesekette wird offensichtlich durch Induktion und Repression eines oder mehrerer der daran beteiligten Enzyme gesteuert. Die Synthese beginnt sofort, wenn der $\mathrm{O}_{2}$-Partialdruck im Medium einen kritischen Wert unterschreitet und wird ebenso rasch gestoppt, wenn die $\mathrm{O}_{2}$-Konzentration wieder ansteigt ${ }^{9,13}$. Die Induktion führt zu keiner Synthese, wenn die DNS, m-RNS- oder die Enzymproteinbildung durch Mitomycin, Actinomycin ${ }^{4,5}$ bzw. Puromycin gehemmt werden. Es scheint sicher, daß neben diesem noch andere Mechanismen an der Regulation beteiligt sind. So hat die Lichtintensität einen Einfluß auf die B.-Chlorophyllkonzentration der Thylakoide und auf die Thylakoidmenge pro Zelle ${ }^{8,11,12,13}$. Die auch bei höheren Organismen beobachtete Kopplung zwischen Strukturprotein und B.-Chlorophyllsynthese ist bei Zusatz oder Änderung der Konzentration von verschiedenen Faktoren (Licht, Actinomycin, Mitomycin, Puromycin, Chloramphenicol, $\mathrm{O}_{2}$-Konzentration, Aminosäuren) nachgewiesen worden und spricht für eine gemeinsame Regelung der Strukturgene. Auch die bisher an phototroph negativen Mutanten gemachten Erfahrungen lassen sich in dieses Schema einordnen.

Die Deutsche Forschungsgemeinschaft unterstützte die Untersuchungen durch eine Sachbeihilfe. Fräulein M. Schäfer und Fräulein R. NeumanN danke ich für ihre interessierte Mitarbeit. 